\title{
INJECTION PAINTING OPTIMIZATION WITH FUZZY LOGIC EXPERT SYSTEM *
}

\author{
J. Beebe-Wang and J. Tang, Brookhaven National Laboratory, Upton, NY 11973, USA
}

\section{Abstract}

Optimizing transverse particle distributions in the accumulator ring is one of most important factors to the future performance of the Spallation Neutron Source (SNS) [1]. This can only be achieved by optimizing the injection bumps that paint the beam in phase space. The process is complex due to the vague distribution inputs and the multiple optimization goals. Furthermore, the priority of the optimization criteria could change at different operational stages. We propose optimizing transverse phase space painting with fuzzy logic and present our initial studies toward that end. The focus of this paper is on how the problem can be solved with a Fuzzy Logic (FL) expert system through the creation of a set of rules that can be applied by the system. Various particle distributions, from computer simulations, are analyzed with FL and the results are compared and discussed. Finally, a run-time optimization control system is proposed.

\section{INTRODUCTION}

Injection painting is a multi-turn injection process with a controlled phase space offset between the centroid of the injected beam and the closed orbit in the ring in order to produce the desired transverse beam distributions. The particle distribution is critically dependent on the choice of painting schemes and the motion of injection orbit bumps. In the case of SNS accumulator ring, the longitudinal beam manipulation is decoupled from transverse phase space painting, we can separate the transverse painting from the longitudinal paintings. There are two basic transverse painting schemes, correlated and anti-correlated painting, incorporated in the SNS accumulator ring design [2]. We choose the correlated painting scheme for this investigation because correlated painting has a better chance to meet the target requirement and may minimize the halo.

The injection painting optimization is a complex process with the multiple goals. At the different operational stage, the priority of the optimization criteria could be different. In general, the three major criteria are:

(1) Satisfy the target requirements (Table 1);

(2) Reduce beam loss at primary collimator;

(3) Reduce foil-hits, thereby reducing beam losses while maintaining an adequate foil lifetime.

The art of the optimization is to make compromises between the beam distribution requirements imposed by the optimization criteria. An optimized injection bump produces a particle distribution that best meets all of the criteria with the given priorities. Figure 1 illustrate the

*Work performed under the auspices of the U. S. Department of Energy
Table 1: Beam requirements at the target

\begin{tabular}{|l|c|}
\hline Beam horizontal dimension & $200 \mathrm{~mm}$ \\
\hline Beam vertical dimension & $70 \mathrm{~mm}$ \\
\hline $\begin{array}{l}\text { Time-averaged beam current } \\
\text { density over beam footprint }\end{array}$ & $<0.143 \mathrm{~A} / \mathrm{m}^{2}$ \\
\hline $\begin{array}{l}\text { Beam power within target } \\
\text { and outside nominal spot }\end{array}$ & $<10 \%$ \\
\hline $\begin{array}{l}\text { Peak time-averaged beam } \\
\text { current density over } 1 \mathrm{~cm}^{2}\end{array}$ & $\leq 0.25 \mathrm{~A} / \mathrm{m}^{2}$ \\
\hline
\end{tabular}

multiple optimization criteria and their required beam distributions. The first criterion, "meeting the target requirements", requires beam distribution with lower density in the middle and higher density on the edge as the illustration at the left side of figure 1. For example, such a distribution can be produced by an exponential bump function with $\tau=0.3 \mathrm{msec}$. The longer the time constant $\tau$, the higher the density in the middle and the lower the density on the edge. An exponential bump function with $\tau=0.6 \mathrm{msec}$ produces a distribution with higher density in the middle and lower density on the edge as the illustration at the right side of figure 1. Such distribution is desirable by the second criterion "Reduce beam loss at primary collimator" but undesirable by the first criterion. The third criterion, "reducing foil-hitting rate", depends on the details of the bump motion once the over-all characteristics of the bump motion is optimized.

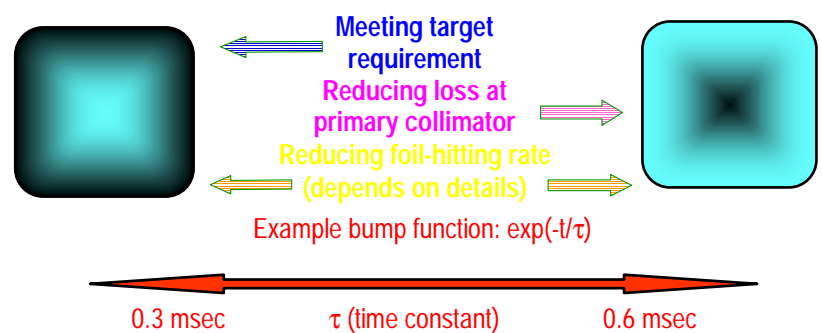

Figure 1 Illustration of the multiple goals of injection painting optimization and the required beam distribution for each goal.

Due to the presence of space charge and magnet errors, there is no simple mathematical model to describe this optimization process. FL provides an alternative problemsolving methodology which mimics the decision making process of a human expert instead of mathematical model [3]. This method can be implemented in hardware, software, or a combination of both. An injection painting optimization system with will benefit upcoming SNS machine commissioning and operations.

In this paper we propose optimizing transverse phase space painting with FL and present our initial findings. In 
section II we show step by step how a FL system can be utilized in the injection painting analysis in order to identify good painting results based on a given set of beam property criteria. In section III, a FL system is applied to simulated injection painting distributions and the system's performance is presented and summarized. In Section IV, a run-time optimization control system with FL is proposed for future SNS operations. Finally, in Section V, the advantages and limitations of using a FL expert system are discussed.

\section{IMPLEMENTING FL TO INJECTION PAINTING}

The key to injection painting optimization is to identify good painting results based on a given set of beam property criteria. In this section we show how this can be achieved by implementing FL with the following seven processing steps.

\section{1 input and output relationships}

First, we need to choose a minimum number of variables for input into the FL engine and determine the input and output relationships. It would seem logical to choose three parameters, which characterize the three criteria listed in Section 1, to be the FL inputs. However, the FL system quickly becomes complex when many inputs and outputs are chosen for a single implementation. Generally, it is advantageous to break the system into smaller pieces and give each limited responsibility. As the first order optimization, our FL system has two inputs, which characterize the first two criteria, and one output which provides a definite conclusion of whether the given injection bump has produced a desirable beam distribution. This FL system can then be extended to include the third input and any other higher order considerations for a further optimization.

\subsection{Data pre-processing}

The first input parameter is the " unevenness" of transverse particle distribution P1 defined as:

$$
P_{1}=\frac{D_{\text {max }}-D_{\text {min }}}{D_{\text {ave }}}
$$

where $D_{\max }, D_{\min }$ and $D_{\text {ave }}$ are maximum and minimum local particle densities and the average beam density, respectively. The second input parameter is the "emittance growth" due to space charge $P_{2}$ defined as:

$$
P_{2}=\frac{\left(\varepsilon_{S C}-\varepsilon_{N S C}\right) \varepsilon_{S C}}{\varepsilon_{120}^{2}}
$$

where $\varepsilon_{S C}$ is the full (99.9\%) final beam emittance obtained from beam profile monitor or computer simulations with space charge, $\varepsilon_{N S C}$ is the full (99.9\%) final beam emittance obtained from computer simulations under the identical condition without space charge, and $\varepsilon_{120}=120 \pi \mathrm{mm}-\mathrm{mr}$ is used to normalize $P_{2}$ to the design full emittance.

\subsection{Fuzzification Process}

The fuzzification process is achieved by introducing the concept of "fuzzy variables". The fuzzy inputs are the nouns, "unevenness" and "emittance growth". The fuzzy variables are adjectives that modify the inputs. We choose "perfect", "good", "ok" and "bad" in our basic FL system. Additional ranges could be used to extend the responsiveness to exceptional or very nonlinear conditions.

\subsection{Establishing Rule Matrix}

Once a set of input fuzzy variables are defined, we need to determine the desired system output response by breaking the problem down into a series of "IF X AND Y THEN" rules. The "rule matrix" is a simple graphical tool for mapping the rules of the FL system. In our basic

\begin{tabular}{|c|c|c|c|c|}
\hline Results & Perfect & Good & $\mathrm{Ok}$ & $\mathrm{Bad}$ \\
\hline Perfect & Perfect & Good + & $\underline{O k+}$ & $\underline{B a d}$ \\
\hline Good & Good + & Good & $\underline{O k}$ & $\underline{\mathrm{Bad}}$ \\
\hline $\mathrm{Ok}$ & $\underline{O k+}$ & $\underline{O k}$ & $\underline{O k}$ & $\underline{B a d}$ \\
\hline $\mathrm{Bad}$ & $\underline{B a d}$ & $\underline{\mathrm{Bad}}$ & $\underline{B a d}$ & $\underline{B a d}$ \\
\hline
\end{tabular}
system, a 4-by-4 matrix is established as show in fig. 2 .

Fig. 2 the illustration of the 4-by-4 rule matrix of the basic FL system. The top row and left column are inputs for uneveness $(P 1)$ and emittance growth $(P 2)$, respectively.

\subsection{Creating FL Membership functions}

The membership function is a graphical representation of the magnitude of participation of each input. It associates a weight with each of the inputs that are processed, defines functional overlap between inputs, and ultimately determines an output response.

\subsection{Inference Process}

An inference process is needed on the logical product of each rule in order to arrive at combined magnitude for each output membership function. The conclusions are combined to form logical sums. These conclusions feed into the inference process where each response output member function's firing strength is determined.

\subsection{Tuning and System Enhancement}

The last step is to test the system, evaluate the results, tune the rules and membership functions, and retest until satisfactory system response are obtained. Tuning the system can be done by changing the rule antecedents or conclusions, changing the centers of the input and/or output membership functions, or adding additional degrees to the input and/or output functions and output response. 


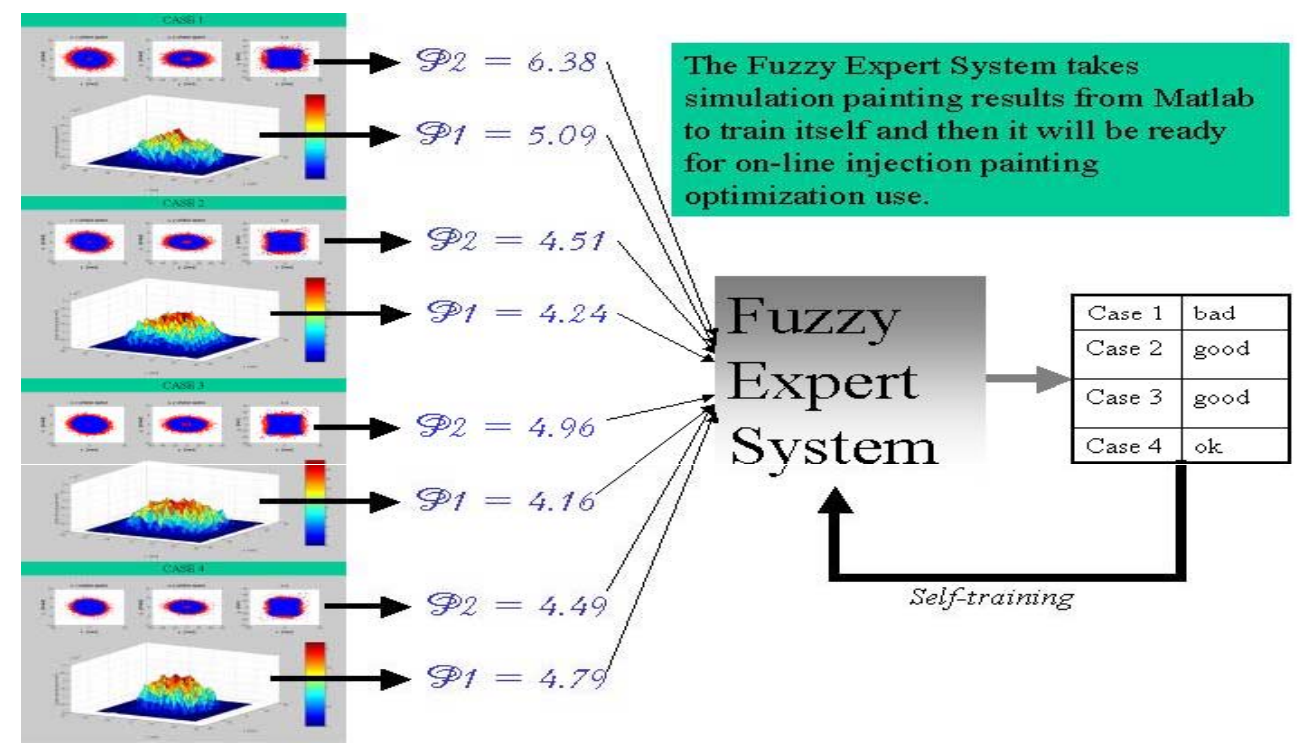

Figure 3. Case studies with FL expert system.

\section{RESULTS}

During future operations the injection input parameters will be provided to the FL system by a beam profile monitor in a pre-process step. Before the on-line data becoming available, we test the FL system with the data from simulations. Figure 3 shows the initial results of 4 cases with the prototype FL expert system. These results have reached good agreement with the judgments of a human expert.

\section{A RUN-TIME EXPERT SYSTEM}

A prototype run-time FL expert system for injection painting optimization is currently under the development. The Experimental Physics and Industrial Control System (EPICS) [4] and Matlab Fuzzy Logic ToolBox [5] are used as tools for the implementation. The SNS global control system is based on EPICS. Figure 4 shows the diagram of the on-line FL expert system and figure 5 shows the prototype system being implemented with EPICS and Matlab tools. More work needs to be done before the system can be employed for the use of the SNS Injection commissioning at ORNL in 2005.

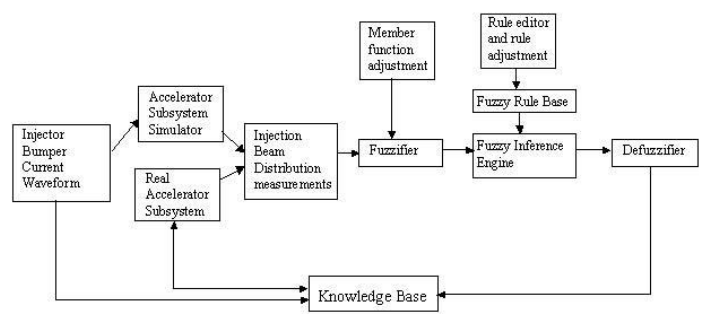

Figure 4. Diagram of the on-line FL expert system

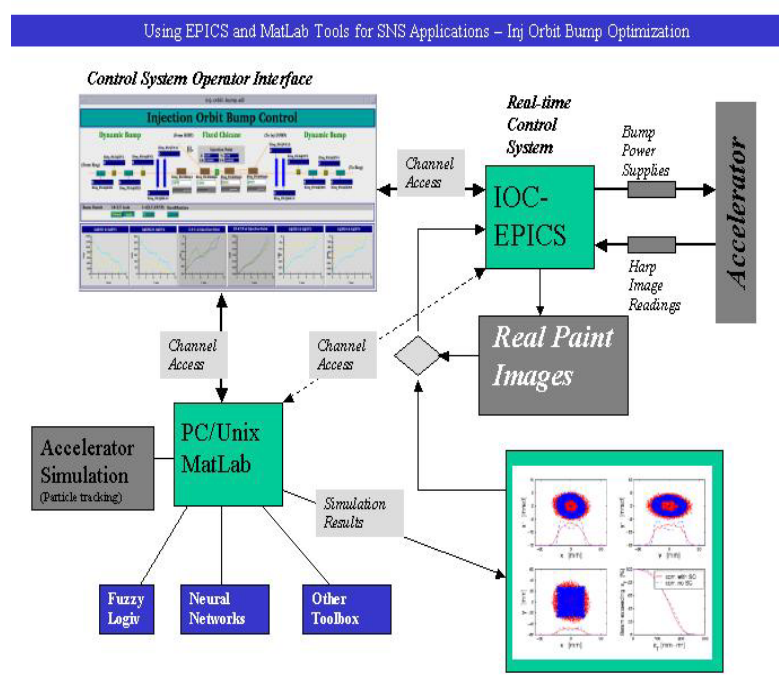

Figure 5 . The prototype system implemented with EPICS [4] and Matlab tools [5].

\section{REFERENCES}

[1] J. Wei et al, "Low-Loss Design for the High-Intensity Accumulator Ring of the Spallation Neutron Source", Accepted for publication in PRST-AB, 2000.

[2] J. Beebe-Wang, Y. Y. Lee, D. Raparia, J. Wei, J., C. R. Prior, and S. Machida, "Beam Properties in the SNS Accumulator Ring Due to Transverse Phase Space Painting", Proceedings of the EPAC 2000, Vienna, Austria, 26-30 June 2000, p.1465-1467.

[3] J.Y. Tang, et al, "A Comparative Study of Fuzzy Logic and Modern Control with EPICS", ICALEPCS'95, Chicago, (1995)

[4] L.R. Dalesio, et al.,"EPICS Architecture",Nuclear Instruments \& Methods in Physics Research A 352 (1994), 197-184

[5] The MatWorks, Inc. http://www.mathworks.com/ 